This item was submitted to Loughborough's Research Repository by the author.

Items in Figshare are protected by copyright, with all rights reserved, unless otherwise indicated.

\title{
Body mapping of cutaneous wetness perception across the human torso during thermo-neutral and warm environmental exposures
}

\section{PLEASE CITE THE PUBLISHED VERSION}

https://doi.org/10.1152/japplphysiol.00535.2014

\section{PUBLISHER}

American Physiological Society

\section{VERSION}

AM (Accepted Manuscript)

\section{PUBLISHER STATEMENT}

This work is made available according to the conditions of the Creative Commons Attribution-NonCommercialNoDerivatives 4.0 International (CC BY-NC-ND 4.0) licence. Full details of this licence are available at: https://creativecommons.org/licenses/by-nc-nd/4.0/

\section{LICENCE}

CC BY-NC-ND 4.0

\section{REPOSITORY RECORD}

Filingeri, Davide, Damien Fournet, Simon Hodder, and George Havenith. 2014. "Body Mapping of Cutaneous Wetness Perception Across the Human Torso During Thermo-neutral and Warm Environmental Exposures". Loughborough University. https://hdl.handle.net/2134/16590. 
1 Body mapping of cutaneous wetness perception across the human torso during thermoneutral and warm environmental exposures

$5 \quad{ }^{1}$ Environmental Ergonomics Research Centre, Loughborough Design School, Loughborough

6 University, LE11 3TU, Loughborough, United Kingdom

$7 \quad{ }^{2}$ Thermal Sciences Laboratory, Oxylane Research, 59665, Villeneuve d'Ascq, France

9 Running title: REGIONAL DIFFERENCES IN CUTANEOUS WETNESS PERCEPTION

11 Correspondence:

12 Davide Filingeri

13 Environmental Ergonomics Research Centre, Loughborough Design School, Loughborough 14 University, LE11 3TU, Loughborough, United Kingdom

15 Phone: $+44(0) 1509223022$

16 E-mail: D.Filingeri@lboro.ac.uk,

18 Author contributions:

19 Davide Filingeri, Damien Fournet, Simon Hodder and George Havenith conception and 20 design of research; Davide Filingeri performed experiments and analysed data; Davide 21 Filingeri and George Havenith interpreted results of experiments; Davide Filingeri prepared 22 figures and drafted manuscript; Davide Filingeri, Damien Fournet, Simon Hodder and 23 George Havenith edited and revised manuscript; Davide Filingeri, Damien Fournet, Simon 24 Hodder and George Havenith approved final version of manuscript. 


\section{ABSTRACT}

27 Sensing skin wetness is linked to inputs arising from cutaneous cold-sensitive afferents. As 28 thermosensitivity to cold varies significantly across the torso, we investigated whether similar 29 regional differences in wetness perception exist. Also, we investigated the regional 30 differences in thermal pleasantness and whether these sensory patterns are influenced by 31 ambient temperature. Sixteen males $(20 \pm 2 \mathrm{yr})$ underwent a quantitative sensory test under 32 thermo-neutral $\left(\mathrm{T}_{\mathrm{air}}=22^{\circ} \mathrm{C} ; \mathrm{RH}=50 \mathrm{p} \cdot 100\right)$ and warm conditions $\left(\mathrm{T}_{\mathrm{arr}}=33^{\circ} \mathrm{C} ; \mathrm{RH}=50 \mathrm{p} \cdot 100\right)$.

33 Twelve regions of the torso were stimulated with a dry thermal probe $\left(25 \mathrm{~cm}^{2}\right)$ with a 34 temperature of $15^{\circ} \mathrm{C}$ below local skin temperature $\left(T_{\text {sk }}\right)$. Variations in $T_{\text {sk }}$, thermal, wetness 35 and pleasantness sensations were recorded. As a result of the same cold-dry stimulus, the skin 36 cooling response varied significantly by location $(p=0.003)$. The lateral chest showed the 37 greatest cooling $\left(-5 \pm 0.4^{\circ} \mathrm{C}\right)$ while the lower back the smallest $\left(-1.9 \pm 0.4^{\circ} \mathrm{C}\right)$. Thermal 38 sensations varied significantly by location and independently from regional variations in skin 39 cooling with colder sensations reported on the lateral abdomen and lower back. Similarly, the 40 frequency of perceived skin wetness was significantly greater on the lateral and lower back as 41 opposed to the medial chest. Overall wetness perception was slightly higher under warm 42 conditions. Significantly more unpleasant sensations were recorded when the lateral abdomen 43 and lateral and lower back were stimulated. We conclude that humans present regional 44 differences in skin wetness perception across the torso, with a pattern similar to the regional 45 differences in thermosensitivity to cold. These findings indicate the presence of a 46 heterogeneous distribution of cold-sensitive thermo-afferent information.

48 KEYWORDS: skin wetness, temperature, body mapping, thermoreceptors, pleasure 


\section{INTRODUCTION}

50 Thermosensitivity (i.e. the ability to perceive thermal changes in the surrounding

51 environment) represents an important drive of thermoregulatory responses in humans and in 52 other mammalian and non-mammalian species $(23,54)$. In humans, cutaneous

53 thermosensitivity is peripherally sub-served by cold-sensitive, myelinated A $\delta$-nerve fibers

54 (conduction velocities ranging from 5-30 m/s $\mathrm{m}^{-1}$ and by cold- and warm-sensitive, 55 unmyelinated C-nerve fibers (conduction velocities ranging from $\left.0.2-2 \mathrm{mss}^{-1}\right)(11,48)$ and 56 centrally integrated by the primary and secondary somatosensory cortices as well as the 57 insular cortex (a cortical region involved in cold and warm temperatures sensation, as well as 58 pain and touch) (15) through the spino-thalamic tract and the the dorsal-column medial 59 lemniscal pathway (36). Fluctuations in skin temperature $\left(\mathrm{T}_{\text {sk }}\right)$ due to environmental stimuli 60 [e.g. changes in ambient temperature $\left(\mathrm{T}_{\text {air }}\right)$ and humidity $\left.(\mathrm{RH})\right]$ and the related thermal 61 sensations have been shown to trigger autonomic (e.g. vasomotor tone and 62 sweating/shivering response) $(32,51)$ and behavioral responses (e.g. adding or removing 63 clothing) (50). These responses aim to maintain thermal homeostasis and comfort $(8,49)$.

64 Despite the critical role of thermosensitivity, sensing temperature is not the only factor 65 amongst the cutaneous sensory afferent to contribute to thermoregulatory responses in 66 humans. Sensing cutaneous wetness is also critical both for behavioral and autonomic 67 responses. Perceiving changes in both ambient humidity and skin wetness have been shown 68 to impact thermal comfort (22) and thus the thermoregulatory behavior (49), both in healthy 69 and clinical populations (e.g. individuals suffering from rheumatic pain) (55). From an 70 autonomic perspective, the degree of skin wetness influences sweat gland function through a 71 progressive suppression of the sweat output (i.e. hidromeiosis) in the presence of wetted skin 72 (38). This results in a reduced ability to lose heat to the environment via evaporative cooling, 73 potentially affecting the thermal balance of the body (12). However, although the ability to 
74 sense skin wetness plays an important role in several behavioral and thermophysiological

75 functions, little it is known on how skin wetness is sensed in humans (37).

76 As opposed to insects, in which humidity receptors sub-serving hygrosensation have been

77 identified and widely described (57), humans seem not to be provided with specific receptors

78 for the sensation of wetness (13). Thus, we seem to "learn" to perceive the wetness

79 experienced when the skin is in contact with a wet surface or when sweat is produced (6)

80 through a complex multisensory integration (17) of thermal (i.e. heat transfer) and tactile (i.e.

81 mechanical pressure and skin friction) inputs generated by the interaction between skin, 82 moisture and (if donned) clothing (22). This hypothesis has been supported by our previous

83 findings. We have recently demonstrated that an illusion of local skin wetness can be evoked

84 during the skin's contact with a cold-dry surface producing skin cooling rates in a range of

850.14 to $0.41{ }^{\circ} \mathrm{C}^{-1}(19,21)$, a temperature course which is similar to the one suggested to 86 occur when the skin is physically wet (16). This could be due to the fact that we seem to 87 interpret the coldness experienced during the evaporation of moisture from the skin as a 88 signal of the presence of moisture (and thus wetness) on the skin' surface. In line with this 89 hypothesis, we have also observed that during the static contact with a warm-wet surface 90 (with a temperature warmer than the skin) no local skin wetness was perceived, as no skin 91 cooling, and thus no cold sensations occurred (20). Finally, this concept has been further 92 confirmed in our most recent study, in which we observed that, when participants' cold 93 sensitivity was significantly reduced through a compression ischemia protocol, skin wetness 94 perception in response to a wet stimulus was also significantly reduced both on hairy and 95 glabrous skin sites (18). All in all, these recent findings have highlighted the critical role of 96 thermosensitivity to cold in the ability to perceive skin wetness (18-21).

97 Appraising the importance of cold afferents in the ability to sense cutaneous wetness has led 98 us to hypothesize that regional differences in wetness perception might exist across the body 
99 and might depend upon the regional differences in thermosensitivity to cold. The distribution 100 of cutaneous sensitivity to cold has been indeed repeatedly shown to vary significantly 101 across different regions of the body $(7,31,39)$ as well as within the same body region (44).

102 For example, the torso is suggested as amongst the most sensitive regions to cold $(7,31,39)$.

103 In this regard, the recent work of Ouzzahra et al. (2012) (44) has provided evidence for the 104 presence of an uneven distribution of cold sensitivity across the front and back torso. If we 105 accept the hypothesis that sensing skin wetness is primarily driven by the level of coldness 106 experienced, it is reasonable to hypothesize that wetness perception varies significantly 107 across the torso, with a pattern which could be similar to the one of thermosensitivity to cold. 108 To our knowledge, only few studies have investigated whether humans present regional 109 differences in cutaneous wetness perception $(2,22,34)$.

110 In a study in which thermal comfort sensitivity was investigated in relation to locally 111 manipulated skin wetness (as resulting from sweat production), Fukazawa and Havenith 112 (2009) (22) found that the torso seems to have a lower sensitivity to wetness than the limbs, 113 while in a non-manipulated condition (natural wetness distribution across the torso) Gerrett et 114 al. (2013) (25) showed that the torso seemed to dominate wetness perception. Similarly, Lee 115 et al. (2011) (34) showed that when asked, individuals reported the torso (i.e. chest and back) 116 to be the region more often perceived as wet during rest and moderate exercise in 25 and $11732{ }^{\circ} \mathrm{C} \mathrm{T}_{\text {air }}$ and 50 p.100 RH. In line with Lee et al. (2011) (34), Ackerley et al. (2012) (2) 118 have recently shown that when wet stimuli with different moisture contents (range: 20-160 $119 \mu \mathrm{L}$ over a $0.0024 \mathrm{~m}^{2}$ surface) were applied to different body regions, individuals were able to 120 differentiate between moisture levels, with a tendency of the back as being amongst the most 121 sensitive region to wetness. The outcomes of these studies have provided initial insights 122 about the regions on which skin wetness might be perceived to a larger extent (e.g. the torso). 123 However, by only measuring the physical wetness (whether due to sweat production or to 
124 contact with a wet surface) these studies have failed to provide a link between the thermal 125 changes occurring locally at the skin's surface when this is wet [variation in local $\mathrm{T}_{\mathrm{sk}}\left(\Delta \mathrm{T}_{\mathrm{sk}}\right)$ ], 126 and how these are perceived in terms of thermal sensations and perception of skin wetness.

127 The aim of the present study was to investigate the regional distribution of skin wetness 128 perception across the torso, in relation to the distribution of thermosensitivity to cold. Also, 129 as local thermal sensations resulting from the same thermal stimulation have been shown to 130 change according to the body's thermal state (e.g. greater cold sensitivity can be observed 131 during heat exposure) $(4,8,21)$, we investigated whether the regional distribution of skin 132 wetness perception is influenced by the environmental conditions (thermo-neutral vs. warm). 133 Finally, as it has been previously suggested that the hedonic attribute (i.e. pleasure) of a 134 thermal stimulus is dependent on the perception of the actual thermal state of the body (e.g. if 135 the direction of the thermal stimulus is oriented towards a shift in the thermal state of the 136 body from its natural homeostasis, then this will result in thermally unpleasant sensations) (4, 137 9), we investigated whether regional differences in thermal pleasantness in response to local 138 skin cooling exist across the torso.

139 We tested the hypothesis that during the short contact with the same cold-dry stimulus (i.e. $14015^{\circ} \mathrm{C}$ lower than local $\mathrm{T}_{\text {sk }}$ ) which we have previously shown to induce an illusion of skin 141 wetness (21), local $\mathrm{T}_{\mathrm{sk}}$, thermal and wetness sensations will vary significantly by location of 142 stimulation. Regions with a high thermosensitivity to cold were expected to present a higher 143 perception of skin wetness. Also, we hypothesized that, as local thermal sensations resulting

144 from the same thermal stimulation have been shown to change according to the body's 145 thermal state $(4,8)$, thermal and wetness perceptions will be higher during a warm as 146 opposed to a thermo-neutral environmental exposure. This was also hypothesized to impact 147 the hedonic component of thermal stimulation (i.e. greater displeasure will be recorded 148 during thermo-neutral as opposed to warm exposure), with regional differences in thermal 
149 pleasure/displeasure expected to follow a pattern similar to the one for thermosensitivity to 150 cold.

151

152

MATERIALS AND METHODS

153

154 Participants

155 Sixteen healthy Caucasian male students (age $20 \pm 2 \mathrm{yr}$; height $1.78 \pm 0.10 \mathrm{~m}$; body mass $15677.4 \pm 10 \mathrm{Kg}$; body composition by skinfold analysis $8.0 \pm 3$ p.100 body fat) with no history 157 of sensory-related disorders volunteered to participate in this study.

158 To account for the inter-individual variability in the hairiness of the torso, participants' hair 159 growth was visually graded using a modified Garn (1951) (25) scoring system (for an 160 extensive review see Yildiz et al. 2010) (58). Photos of the front and back torso of each 161 participant were taken. A score of 0-4 was assigned to chest, abdomen and upper and lower 162 back, based on the visual density of terminal hairs. A score of 0 represented the absence of 163 terminal hairs, a score of 1 minimally evident hair growth, and a score of 4 extensive hair 164 growth (58). Thirteen out of 16 participants presented minimal hairs on the chest (score $=0.2$ $165 \pm 0.1)$ and abdomen (score $=0.3 \pm 0.1)$ and the absence of terminal hairs on the upper and 166 lower back. Three out of 16 participants presented a higher level of hairiness on the chest $167($ score $=3 \pm 0.6)$ and abdomen $($ score $=2.3 \pm 0.3)$ and the absence of hairs on the upper and 168 lower back.

169 All participants gave their informed consent for participation. The test procedure and the 170 conditions were explained to each participant. The study design had been approved by the 171 Loughborough University Ethics Committee and testing procedures were in accordance with 172 the tenets of the Declaration of Helsinki. 


\section{Experimental Design}

175 All participants underwent the same quantitative sensory test under thermo-neutral $\left(\mathrm{T}_{\text {air }}=\right.$ $17622^{\circ} \mathrm{C} ; \mathrm{RH}=50$ p.100) and warm environmental conditions $\left(\mathrm{T}_{\text {air }}=33^{\circ} \mathrm{C} ; \mathrm{RH}=50 \mathrm{p} .100\right)$. The 177 quantitative sensory test was based on the application of a cold-dry stimulus on 12 different 178 skin sites distributed across the front and back torso of each participant. The exact anatomical 179 locations of the areas targeted for stimulation are described in figure 1 and are in line with the 180 work of Ouzzahra et al. (2012) (44). All tested sites were medial or on the left side of the 181 body, assuming symmetry (14). During the contact with the stimulus, participants reported 182 their local thermal, wetness and pleasantness sensations on Likert scales. Local $\mathrm{T}_{\text {sk }}$ at the 183 contact site was measured before and immediately after the contact with the stimulus using a 184 single spot infrared thermometer (FLUKE 566, Fluke Corporation, USA) with a temperature 185 range of -40 to $800{ }^{\circ} \mathrm{C}$ and an accuracy of $\pm 1{ }^{\circ} \mathrm{C}$. In order to maximize the accuracy of the 186 temperature readings, during each test the infrared thermometer was calibrated against a 187 black plate whose temperature was monitored with a thermistor (Grant Instruments, 188 Cambridge, UK). This method has been previously used (see Filingeri et al. 2014) (21) and 189 shown to be effective in allowing recording of post-stimulation $\mathrm{T}_{\mathrm{sk}}$ to be made consistently 190 close to the when subjective sensations were rated. The cold-dry stimulus was delivered by a 191 square thermal probe (Physitemp Instruments Inc., USA) with a contact surface of $0.0025 \mathrm{~m}^{2}$.

192 The relative temperature of the stimulus was $15^{\circ} \mathrm{C}$ lower than the local $\mathrm{T}_{\text {sk }}$ which was 193 measured with the infrared thermometer. We chose a relative temperature of $-15^{\circ} \mathrm{C}$ as we 194 have previously shown this to evoke the highest levels of perceived wetness during a 10-s 195 contact with the upper and lower back of resting and exercising individuals (21).

196 A single-blind psychophysical approach was used for this study. Participants were informed 197 only about the body region objected to the stimulation, and no information was provided on 198 the type and magnitude of the stimulation to limit any expectation effects. To assure that the 
199 participants could not see the stimulus applied on their torso, the following set up was

200 designed. When the front torso was stimulated, participants were asked to lie on a bench on 201 their back, with their arms alongside the body and a rectangular-shaped textile screen (length: $2020.8 \mathrm{~m}$; height: $0.7 \mathrm{~m}$ ) was placed above participants' neck. The screen was adjusted until each 203 participant confirmed that they could not see either their front torso or the investigator. When 204 the back torso was stimulated, participants were asked to lie on their front, with their arms 205 alongside the body, and to face towards the left, while the investigator was standing on their 206 right hand side. Each participant confirmed that they could not see either their back torso or 207 the investigator. The 12 skin sites were stimulated on a balanced order to prevent any order 208 effect. The data collection took place in December (mean monthly temperature: $5.1{ }^{\circ} \mathrm{C}$; min209 max temperature range: 2.0 to $8.2{ }^{\circ} \mathrm{C}$ ).

210

\section{Experimental Protocol}

212 Participants arrived at the laboratory $30 \mathrm{~min}$ before the time scheduled for the test to allow 213 preparation procedures. First, semi-nude body mass, height and skinfolds thickness (seven

214 sites) were measured and recorded. For body composition calculations ACSM's guidelines 215 for exercise testing and prescription were used (27). Body density was calculated using the 216 following seven sites (chest, midaxillary, triceps, subscapular, abdomen, suprailiac and thigh) 217 equation:

$$
\begin{aligned}
\text { Body density } & =1.112-0.00043499(\text { sum of seven skinfolds }) \\
& +0.00000055(\text { sum of seven skinfolds })^{2}-0.00028826(\text { age })
\end{aligned}
$$

219 Participants then changed into shorts, socks and running shoes. Five iButtons (Maxim, USA) 220 were taped to five skin sites on the right side of the body (i.e. cheek, abdomen, upper arm, 221 lower back and back lower thigh) to record local $\mathrm{T}_{\mathrm{sk}}$. The five temperature measurements 
222 were recorded at $1 \mathrm{~min}$ intervals throughout the tests, averaged every $5 \mathrm{~min}$, and then 223 weighted according to the work of Houdas and Ring (1982) (29) to give an estimate of mean $224 \mathrm{~T}_{\mathrm{sk}}$ for the entire body. The 12 skin sites targeted for stimulation were marked with a 225 washable marker to assure consistency in the location of stimulation.

226 After preparation, participants entered a first environmental chamber set for the thermo227 neutral exposure $\left(22{ }^{\circ} \mathrm{C} \mathrm{T}\right.$ air, 50 p.100 RH). Participants sat on a chair and waited 10 min to 228 allow acclimation to the environmental conditions. During this period, participants were 229 familiarized with the rating scales designed to record individual thermal, wetness and 230 pleasantness sensations: an 11 point thermal scale (-6 very cold; -4 cold; -2 slightly cool; 0 231 neutral; +2 slightly warm; +4 warm); an 11 point wetness scale (-6 dripping wet; -4 wet; -2 232 slightly wet; 0 neutral; +2 slightly dry; +4 dry); an 11 point pleasantness scale (-6 very 233 unpleasant; -4 unpleasant; -2 slightly unpleasant; 0 neutral; +2 slightly pleasant; +4 pleasant) $234(21,43)$. No descriptors were applied to intermediate scores (i.e. $-5 ;-3 ;-1 ;+1 ;+3)$. We 235 defined the value -2 (labelled: "slightly wet") of the wetness scale as our set threshold to 236 identify a clearly perceived local skin wetness.

237 After the acclimation period and according to the order of stimulation, participants were 238 asked to lie either on their front or back and the quantitative sensory test was initiated. 239 Participants were first asked to rate their thermal and wetness sensations only, just before the 240 application of the stimulus (i.e. baseline whole-body sensation), while the local $\mathrm{T}_{\mathrm{sk}}$ of the 241 skin site targeted for stimulation was measured with the infrared thermometer. Then the 242 thermal probe was set to the required relative temperature (i.e. $15^{\circ} \mathrm{C}$ below the recorded local $243 \mathrm{~T}_{\mathrm{sk}}$ ) and applied by hand to the skin site. To avoid an effect of surprise on the transient 244 sensations, a verbal warning was given prior to stimulation. The application of the probe 245 consisted of a short contact lasting 10s. During the stimulation, the probe was not moved and 246 participants could not see the stimulated area. At the end of the $10 \mathrm{~s}$ stimulation, participants 
247 were instructed and encouraged to verbally report their local thermal, wetness and also

248 pleasantness sensations, using whatever number in the scales seemed appropriate (integers

249 only). Immediately after this the probe was removed and $\mathrm{T}_{\mathrm{sk}}$ of the stimulated area was

250 recorded with the infra-red thermometer. The same protocol was repeated for each of the 12

251 skin sites allowing at least one minute in between them. Each participant had only one

252 presentation of each stimulus for each skin site. The quantitative sensory test lasted for $15 \mathrm{~min}$.

253 After completion of the test, 10min were allowed before participants moved from the first to

254 the second environmental chamber set for the warm exposure $\left(33{ }^{\circ} \mathrm{C} \mathrm{T}_{\text {air }}, 50 \mathrm{p} .100 \mathrm{RH}\right)$.

255 Once in the second chamber, 10min were allowed for acclimation before the same 256 quantitative sensory test, as explained above, was performed.

257

258 Statistical Analysis

259 In the present study, the independent variables were the skin site stimulated and the 260 environmental condition. The dependent variables were mean, local $\mathrm{T}_{\mathrm{sk}}, \Delta \mathrm{T}_{\mathrm{sk}}$ (i.e. variation 261 from pre- to post-stimulation) and thermal, wetness and pleasantness sensation. All data 262 were first tested for normality of distribution and homogeneity of variance using Shapiro263 Wilk and Levene's tests, respectively.

264 Mean $\mathrm{T}_{\mathrm{sk}}$ data for the thermo-neutral and warm exposure were compared using a paired t-test. 265 Local $\Delta \mathrm{T}_{\text {sk }}$ data were analysed by a 2-way repeated measures analysis of variance, with skin 266 site stimulated (12 levels) and environmental condition (2 levels: thermo-neutral and warm) 267 as repeated measures variables. Data were tested for sphericity and if the assumption of 268 sphericity was violated, Huynh-Feldt or Greenhouse-Geisser corrections were undertaken to 269 adjust the degrees of freedom for the averaged tests of significance. Estimated marginal 270 means and 95 p.100 confidence intervals were used to investigate the main effects and 
271 interactions of the variables. Observed power was computed using $\alpha=0.05$. When a

272 significant main effect was found, Tukey's post-hoc analyses were performed.

273 As absolute thermal, wetness and pleasantness sensations were obtained in the form of

274 ordinal ratings, these were analysed by means of non-parametric statistics. The main effect of

275 the environmental condition (2 levels of comparison) was tested by a Wilcoxon signed rank

276 test $(Z)$ whereas the main effect of the skin site stimulated (12 levels of comparison) was

277 tested by a Friedman's analysis of variance $\left(\mathrm{X}^{2}\right)$. Post-hoc analyses for the effect of skin site

278 stimulated were performed by a Wilcoxon signed rank test $(Z)$ and adjusted for multiple

279 comparisons. Effect size was calculated and reported as $r$. Although the authors acknowledge

280 that non-parametric statistics tend to have less power for well distributed dependent variables,

281 they can be more sensitive to effects when variables are not normally distributed, as in the

282 case of this study (3).

283 To further investigate the regional distribution of cutaneous wetness perception, a frequency

284 distribution analysis of skin wetness was performed. Wetness perception scores as recorded 285 during both environmental conditions were collapsed over the skin site stimulated. Then, as

286 the value -2 of the wetness scale (labelled: "slightly wet") was defined as our set threshold to

287 identify a clearly perceived local wetness, wetness scores from -2 (i.e. "slightly wet") to -6

288 (i.e. "dripping wet") were grouped and considered as referring to a clear perception of 289 wetness ("wet"), whereas any score in between -1 and +4 (i.e. "dry") was considered as 290 representing no perception of wetness (“dry”). At this point, the frequency of times (p.100) 291 the cold-dry stimulus was perceived as "dry" or as "wet" was calculated and analysed by a 292 Chi-square test. This analysis was performed for each of the 12 skin sites. Also, frequency 293 data were grouped and compared between the front and back torso. The same frequency 294 distribution analysis of wetness ratings has been performed in one of our recent studies (see 295 Filingeri et al., 2014) (21). Also, a similar frequency distribution analysis of thermal ratings 
296 has been previously reported in the literature (see Gan et al., 2012) (24). In line with Gan et 297 al. (2012) (24) and with our previous findings (18), we believe that, because of the variable 298 nature of subjective responses, reorganizing the collected data in this format would make the 299 potential differences in the regional distribution of wetness perception across the torso easier 300 to identify.

301 Finally, a Spearman's rank correlation coefficient was calculated to investigate the degree of 302 association between: 1. thermal sensation and frequency of wetness perception; 2 . 303 pleasantness sensation and frequency of wetness perception; 3. thermal sensation and 304 pleasantness sensation. Statistical analysis was performed using IBM SPSS Statistics 19 305 (IBM, USA). In all analyses, $p<0.05$ was used to establish significant differences. Parametric 306 and non-parametric (perceptual scores) data are reported as mean \pm standard error of the 307 mean. Furthermore, median and inter-quartile ranges [median; percentile] are reported for 308 non-parametric data.

309

310 RESULTS

311

\section{Mean and local $\mathbf{T}_{\mathbf{s k}}$}

313 Mean $\mathrm{T}_{\mathrm{sk}}$ was calculated for each exposure and found to be normally distributed ( $\left.p>0.05\right)$.

314 Mean $\mathrm{T}_{\text {sk }}$ values for thermo-neutral and warm exposures were respectively $32.4 \pm 0.1^{\circ} \mathrm{C}$ and $31534.8 \pm 0.1{ }^{\circ} \mathrm{C}$. These values were found to be significantly different (mean difference $=2.4{ }^{\circ} \mathrm{C}$; $31695 \mathrm{p} .100 \mathrm{CI}=2.2,2.5{ }^{\circ} \mathrm{C} ; t=36.8$; two tailed $\left.p<0.001\right)$. This result confirms the effectiveness 317 of the environmental conditions we designed in inducing a significant change in the skin's 318 thermal state.

319 Baseline local $\mathrm{T}_{\mathrm{sk}}$ values (pre-stimulation) varied in a range between $31.8 \pm 0.1{ }^{\circ} \mathrm{C}$ (i.e. 320 lateral chest) and $33.4 \pm 0.2{ }^{\circ} \mathrm{C}$ (i.e. medial upper back) for the thermo-neutral exposure, and 
321 between $34.9 \pm 0.2{ }^{\circ} \mathrm{C}$ (i.e. lateral chest) and $36.1 \pm 0.1{ }^{\circ} \mathrm{C}$ (i.e. medial upper back) for the 322 warm exposure. Local $\Delta \mathrm{T}_{\mathrm{sk}}$ (as a result of the relative cold-dry stimulus applied to each skin 323 site during the thermo-neutral and warm exposures), was calculated and found to be normally 324 distributed $(p>0.05)$. The data analysis indicated that only the skin site stimulated had a 325 significant main effect on the local $\Delta \mathrm{T}_{\mathrm{sk}}\left(\mathrm{F}=4.4_{(4.6,50.6)}, p=0.003\right)$. No significant effect of the 326 environmental condition $\left(\mathrm{F}=2.2_{(1,11)}, p=0.17\right)$ nor significant interaction between the skin

327 site stimulated and the environmental condition was found $\left(\mathrm{F}=0.4_{(11,121)}, p=0.4\right)$. The 328 regional distribution of $\Delta \mathrm{T}_{\mathrm{sk}}$ is shown in figure $2 A$. Post-hoc analyses indicated that, 329 depending on skin site, local $\Delta \mathrm{T}_{\text {sk }}$ varied significantly in a range of $-1.9 \pm 0.4{ }^{\circ} \mathrm{C}$ (i.e. medial 330 lower back) to $-5.0 \pm 0.4{ }^{\circ} \mathrm{C}$ (i.e. lateral chest), corresponding to a range of skin cooling rates 331 of $0.19 \pm 0.04$ to $0.5 \pm 0.04{ }^{\circ} \mathrm{C}^{-1} \mathrm{~s}^{-1}$. These values were calculated as the ratio between the $\Delta \mathrm{T}_{\text {sk }}$ 332 from post- to pre-stimulation and the contact time (i.e. 10s). The significance levels are 333 presented separately for sites of the front and back torso (Tab.1).

334 Overall, these outcomes indicated that, as a result of the same relative cold-dry stimulus, the 335 skin cooling response varied significantly by location across the torso, with a pattern which 336 did not change between the thermo-neutral and warm environmental exposure.

\section{Thermal sensation}

339 Baseline thermal sensation scores (pre-stimulation) varied in a range of $0.1 \pm 0.1$ [median=0; $3400.0,1.0]$ to $0.6 \pm 0.2$ [median $=1 ; 1.0,1.0$ ] for the thermo-neutral exposure and of $1.4 \pm 0.3$ $341[$ median $=1 ; 0.2,2.7]$ to $1.7 \pm 0.2[$ median $=2 ; 1.0,2.0]$ for the warm exposure. Expressed in

342 terms of semantic labels, these were in the range of "neutral" for the thermo-neutral exposure 343 and in a range going from "neutral" to "slightly warm" for the warm exposure.

344 In response to the stimuli, thermal sensation scores were overall "less cold" during the warm $345(-3.5 \pm 0.1)[$ median $=-4 ;-4.0,-3.0]$ than during the thermo-neutral exposure $(-3.7 \pm 0.1)$ 
$346[$ median $=-4 ;-5.0,-3.0](\mathrm{Z}=-3.5, p=0.001, \mathrm{r}=-0.25)$. Expressed in terms of semantic labels,

347 these were in a range going from "slightly cool" to "cold" for the warm exposure and in a 348 range going from "slightly cool" to "very cold" for the thermo-neutral exposure. Thermal 349 sensations differed significantly according to the skin site stimulated $\left[X^{2}(11, N=32)=143.2\right.$, $350 p<0.001$ ], with scores varying in a range of $-2.3 \pm 0.2$ [median $=2 ;-3.0,-1.2$ ] (i.e. medial 351 chest) to $-4.4 \pm 0.2$ [median $=4 ;-5.0,-4.0$ ] (i.e. lateral lower back) between sites. Expressed

352 in terms of semantic labels, these were in a range going from "slightly cool" to "very cold".

353 Mean thermal sensations, averaged over both environmental conditions, are shown in figure $3542 B$. The significance levels are presented separately for sites of the front and back torso 355 (Tab.1).

356 Overall, these outcomes indicated that the same relative cold-dry stimulus evoked thermal 357 sensations which were significantly "colder" when the stimulus was applied on specific 358 regions (such as the lateral abdomen and the lateral and lower back) as opposed to other 359 regions (such as the lateral and medial chest), in which the same stimulus evoked "less cold" 360 thermal sensations. Also, the same relative cold-dry stimulus was overall perceived as 361 slightly less cold during the warm than during the thermo-neutral exposure.

362

\section{Wetness perception}

364 Baseline wetness perception scores (pre-stimulation) varied in a range of $0.6 \pm 0.3$ [median= $3650 ; 0.0,2.0$ ] to $1 \pm 0.3$ [median $=0 ; 0.0,2.0$ ] for the thermo-neutral exposure and $0.6 \pm 0.4$ 366 [median $=0 ; 0.0,1.7]$ to $0.8 \pm 0.4$ [median $=1 ; 1.0,2.0]$ for the warm exposure. Expressed in 367 terms of semantic labels, these were in a range going from "neutral" to "slightly dry".

368 In response to the stimuli, local wetness perception scores were overall slightly "wetter" 369 during the warm $(-1.7 \pm 0.1)[$ median $=-2 ;-2.0,-1.0]$ than during the thermo-neutral exposure $370(-1.4 \pm 0.1)[$ median $=-1 ;-2.0,-1.0](\mathrm{Z}=-2.9, p=0.004, \mathrm{r}=-0.2)$. Expressed in terms of 
371 semantic labels, these were in a range going from "neutral" to "slightly wet" for both warm

372 and thermo-neutral exposure. Wetness perceptions differed significantly according to the skin 373 site stimulated $\left[\mathrm{X}^{2}(11, \mathrm{~N}=32)=58.4, p<0.001\right]$, with scores varying in a range of $-1.1 \pm 0.1$

$374[$ median $=-1 ;-1.0,-1.0]$ (i.e. medial chest) to $-2.1 \pm 0.2$ [median $=-2 ;-3.0,-1.0]$ (i.e. medial 375 lower back) between sites. Expressed in terms of semantic labels, these were in a range going 376 from "neutral" to "slightly wet". The significance levels are presented separately for sites of 377 the front and back torso (tab.1). To further investigate the regional distribution of wetness 378 perception, a frequency distribution analysis of wetness scores was performed. The data 379 analysis indicated a main effect of skin site stimulated on the frequency of "wet" scores 380 (Pearson Chi-square $p<0.001$ ). Data for each of the 12 skin sites stimulated are shown in 381 figure $2 C$. The results indicated that the relative cold-dry stimulus was significantly more 382 often perceived as wet when applied to the lower back (lateral $=56$ p. 100 ; medial $=59$ p.100) 383 and the medial upper back (53 p.100). The same stimulus was significantly less often 384 perceived as wet when applied to the medial chest (22 p.100) and medial upper abdomen (28 385 p.100). Overall, the back presented a significantly greater frequency of wetness perception 386 (53 p.100) than the front torso (39 p.100) (Pearson Chi-square $p=0.047)$.

387 Overall, these outcomes indicated that, the same relative cold-dry stimulus evoked wetness 388 perceptions which were significantly "wetter", and more often perceived as wet, when the 389 stimulus was applied on specific regions (such as the medial and lateral lower back) as 390 opposed to other regions (such as the medial and lateral chest), in which the same stimulus 391 evoked "less wet" and less frequent wetness perceptions.

\section{$393 \quad$ Pleasantness sensation}


394 Pleasantness sensations were recorded only during the stimulation as we were primarily 395 interested in the affective and discriminative sensations aroused by the application of the 396 thermal stimulus with regards to the whole body's thermal state.

397 Pleasantness sensation scores were overall "less unpleasant" during the warm $(-1.8 \pm 0.1)$ 398 [median $=-2 ;-3.0,-1.0]$ than during the thermo-neutral exposure $(-2.2 \pm 0.1)$ [median= $-2 ;-$ $3993.0,-1.0](Z=-3.8, p<0.001, r=-0.3)$. Expressed in terms of semantic labels, these were in a 400 range going from "neutral" to "unpleasant" for both the thermo-neutral and warm exposure.

401 Pleasantness sensation scores differed significantly according to the skin site stimulated [ $\mathrm{X}^{2}$ $402(11, \mathrm{~N}=32)=108.1, p<0.001]$, with scores varying in a range of $-1.1 \pm 0.2$ [median $=-1 ;-1.0$, $403-0.2$ ] (i.e. medial chest) to $-2.7 \pm 0.2[$ median $=-2 ;-4.0,-2.0]$ (i.e. lateral lower back). 404 Expressed in terms of semantic labels, these were in a range going from "neutral" to 405 "unpleasant". Mean pleasantness sensations averaged over conditions, as reported during the 406 application of the relative cold-dry stimulus to each skin site, are shown in figure $2 D$.

407 Overall, these outcomes indicated that, the same relative cold-dry evoked sensations which 408 were significantly "more unpleasant" when the stimulus was applied on specific regions 409 (such as the lateral abdomen and lateral lower back) as opposed to other regions (such as the 410 medial chest and medial upper abdomen), in which the same stimulus evoked "less 411 unpleasant" sensations. Interestingly, the regional variation in displeasure showed a pattern 412 similar to the regional distribution in thermosensitivity to cold. Finally, the same relative 413 cold-dry stimulus was overall perceived as slightly less unpleasant during the warm than 414 during the thermo-neutral exposure.

415

416 Correlation analysis between thermal sensation, frequency of perceived wetness and 417 pleasantness sensation 
418 The degree of association between the level of coldness experienced and the frequency of

419 perceived wetness (assessed by a Spearman's rank correlation test) was found to be

420 statistically significant $(p<0.01$; Spearman's rho=0.79), indicating a significant correlation

421 between increasing coldness and increasing frequency of perceived wetness (fig. $3 A$ ).

422 Similarly, the degree of association between the level of pleasantness experienced and the

423 frequency of perceived wetness was found to be statistically significant $(p<0.01$; Spearman's

$424 \mathrm{rho}=0.76$ ), indicating a significant correlation between decreasing pleasantness and 425 increasing frequency of perceived wetness (fig. 3B). Finally, the degree of association 426 between the level of coldness and the level of pleasantness experienced was also found to be 427 statistically significant $(p<0.01$; Spearman's $\mathrm{rho}=0.97)$, indicating a significant correlation 428 between increasing coldness and decreasing pleasantness (fig. 3C).

429

430 DISCUSSION

431 The present study investigated the regional distribution of cutaneous wetness perception 432 across the torso, in relation to the distribution of thermosensitivity to cold. Furthermore, we 433 investigated whether these regional sensory patterns are influenced by different ambient 434 temperatures as well as whether regional differences in thermal pleasantness in response to 435 local skin cooling exist. During a thermo-neutral and warm environmental exposure, by 436 exposing 12 skin sites of the torso to the static contact with the same relative cold-dry 437 stimulus we demonstrated that: 1. cutaneous wetness perception varies significantly across 438 the torso (see fig. 2C), with regions showing high thermosensitivity to cold (e.g. the lower 439 and lateral abdomen and back, see fig. $2 B$ ) presenting wetness perception in larger magnitude 440 and frequency (compare fig. $2 B$ vs. $2 C$ ); 2 . cutaneous wetness perception is slightly higher 441 under warm than under thermo-neutral environmental conditions, despite thermosensitivity to 442 cold appears to be slightly lower; 3. regional variations in thermal pleasure/displeasure exist 
443 across the torso, and show a pattern similar to the regional distribution in thermosensitivity to

444 cold (i.e. greater coldness induced greater displeasure) (compare fig. $2 B$ vs. 2D).

445 In summary, our results indicate that the existence of regional differences in cutaneous

446 thermosensitivity to cold translates into significant regional differences in cutaneous wetness

447 perception across the human torso. Interestingly, these regional sensory patterns were

448 observed to be independent from the magnitude of local skin cooling. In other words, the

449 regions in which the stimulus resulted in greater skin cooling (i.e. lateral chest) were not 450 necessarily the ones in which the stimulus was perceived as colder, wetter and more 451 unpleasant (compare fig. $2 A$ with $2 B, 2 C$ and $2 D$ ). To our knowledge the present study is the

452 first to take into account the regional variation in skin temperature occurring during contact 453 cooling and to link this to the regional distribution of thermosensitivity to cold, skin wetness 454 and thermal pleasure/displeasure across the human torso. The novelty of these findings is in 455 providing the first detailed body maps of thermal, wetness and pleasantness sensation across 456 the human torso.

457

458

The role of thermosensitivity to cold in the ability to sense skin wetness

459 With regards to the role of thermosensitivity to cold in characterizing the ability to sense 460 cutaneous wetness, the outcomes of this study are in line with our previous findings, in which 461 we have demonstrated that the contact with a cold-dry stimulus producing skin cooling rates 462 in a range of 0.14 to $0.41{ }^{\circ} \mathrm{C} \cdot \mathrm{s}^{-1}$ can evoke an illusion of skin wetness $(19,21)$. In the present 463 study, the relative temperature stimulus we used resulted in skin cooling rates ranging from 4640.19 to $0.5{ }^{\circ} \mathrm{C} \cdot \mathrm{s}^{-1}$. Although generated by a dry stimulus, these fluctuations in $\mathrm{T}_{\mathrm{sk}}$ evoked 465 thermal sensations which were associated to the perception of skin wetness, particularly on 466 the back torso. Hence, this finding supports the hypothesis that the central integration of 467 coldness, as primarily sub-served by peripheral myelinated A $\delta$-nerve fibers, is critically 
468 involved in the neural processes underpinning humans' ability to sense wetness $(19,21)$. This

469 proposed theory finds support in a neurophysiological model of skin wetness that we have

470 recently developed (18), which sees the activity of cold afferents being both behaviorally and

471 physiologically necessary to give rise to the perception of wetness. As the skin seems not to

472 be provided with hygroreceptors (13), it is indeed hypothesized that the somatosensory cortex

473 could be involved in generating a neural representation of a "typical wet stimulus". This

474 could be based on the multimodal transformation (i.e. information from one sensory sub-

475 modality can be transformed into a map or reference frame defined by another sub-modality)

476 of the somatosensory inputs generated when the skin is physically wet (28). As the sensory

477 inputs associated to the physical experience of cutaneous wetness are often generated by heat

478 transfer in the form of evaporative cooling (2), the typical neural representation of a wet

479 stimulus might therefore rely on experiencing a certain degree of coldness. This neural

480 representation could be transformed into a firing rate code and then associated to the

481 perception of wetness (46). Hence, when the memorized stimulus (i.e. coldness), as coded by

482 the specific afferents (i.e. A $\delta$-nerve fibers) is presented, wetness will be sensed.

483 The outcomes of this study, in which a cold-dry stimulus evoked an illusion of skin wetness

484 in blindfolded individuals, are in agreement with this sensory model for wetness. However,

485 although the relative temperature stimulus used in this study resulted in skin cooling rates

486 which were within the range suggested to evoke wetness perceptions for all the regions

487 investigated (i.e. 0.19 to $\left.0.5{ }^{\circ} \mathrm{C}^{-1}\right)(16,19,21)$, significant regional variations in wetness

488 perception were observed across the torso. Hence, this indicates that other factors than the

489 degree of local skin cooling (e.g. regional differences in thermal sensitivity and habituation

490 components) might play a significant role in characterizing the cutaneous distribution of

491 wetness perception, at least across the human torso.

492 
494 Within the experimental conditions of this study, the lower back, lateral mid-back and medial 495 upper back, as well as the lateral abdomen presented wetness perception in larger magnitude 496 and frequency than the lateral and medial chest and medial upper abdomen (see fig. 2C). 497 These outcomes are in line with the work of Lee et al. (2011) (34) who have shown the upper 498 and lower back to be most frequently perceived as wet during conditions of sweat-induced 499 physical wetness. Although not statistically significant, a similar trend was observed by 500 Ackerley et al. (2012)(2) who reported the back to present higher wetness perception than 501 other body regions. However, in the mentioned works, no data are reported on any 502 physiological change (e.g. regional differences in $\Delta \mathrm{T}_{\mathrm{sk}}$ ) which could have triggered the 503 sensory inputs used by the participants to discriminate the level of wetness experienced 504 regionally. In the present study, this issue was overcome by quantifing the local $\Delta \mathrm{T}_{\mathrm{sk}}$, 505 recording thermal sensations, and eventually comparing these with the regional distribution 506 of wetness perception. Thus, for the first time we provide evidence in support of the 507 physiological and behavioral significance of the regional differences in cutaneous wetness 508 perception across the torso.

509 In the current study, the local thermal sensations in response to the cold stimulus were 510 observed to be independent from the local $\Delta \mathrm{T}_{\mathrm{sk}}$. A comparison of the body maps of $\Delta \mathrm{T}_{\mathrm{sk}}$ (fig.

$5112 A$ ) and thermal sensation (fig. $2 B$ ) shows that the cold-dry stimulus was perceived as colder 512 when applied to the lower back than to the lateral chest, despite when stimulated, the lower 513 back presented a significantly smaller drop in $T_{\text {sk }}$ than the lateral chest. Interestingly, a 514 similar trend was observed for the perception of wetness (see fig. $2 C$ ). Hence, it could be 515 proposed that, as well as for the thermosensitivity to cold, the regional differences in wetness 516 perception could depend upon an uneven weighting and integration of thermoafferent 517 information, which seems independent from the regional variations in $\mathrm{T}_{\mathrm{sk}}$ and, potentially, 
518 from the density of thermoreceptors $(5,7,39)$. As shown in figures $2 B$ and $2 C$, the regions

519 with high wetness frequency presented a high sensitvity to cold, with the association between

520 the level of experienced coldness and the frequency of perceived wetness being linear (i.e.

521 greater coldness induces more frequent wetness) and statistically significant. Thus, it could be

522 suggested that the sensitvity to coldness (i.e. a neurophysiological variable) rather then local

$523 \Delta \mathrm{T}_{\mathrm{sk}}$ (i.e. a physical variable) might be more critical in characterizing the regional distribution

524 of cutaneous wetness perception. From a neurophysiological point of view, this is in line with

525 what has previously been proposed on the critical role of thermosensitivity to cold in sensing

526 cutaneous wetness $(2,21)$. The higher sensitivity to cold of some regions of the torso could

527 indeed result in these regions being more sensitive to perceive skin wetness. The possibility

528 that colder sensations are more likely to translate in wetter perceptions, is also aligned to the

529 work of Ackerley et al. (2012) (2). In their work, the authors have shown that individuals

530 readily discriminated between very small amount of moisture on the skin (in the range of 40

$531 \mu \mathrm{L}$ over a surface of $0.0024 \mathrm{~m}^{2}$ ). Altough in the mentioned study no recordings of local $\Delta \mathrm{T}_{\text {sk }}$

532 and thermal sensations were performed, in line with the authors, we believe that participants

533 distinguished the greater from the smaller levels of moisture due to the resulting greater

534 evaporative cooling which induced colder thermal sensations.

535 The fact that humans seem to associate "feeling colder" with "feeling wetter" is not entirely

536 surprising, and could be due to learning factors. For example, the contact with a wet surface

537 or the exposure to a cold-humid environment often result in colder sensations than the ones

538 resulting from the contact with a dry surface or the exposure to a cold-dry environment. In

539 this regard, the skin's contact with a wet fabric has been suggested to be perceived as wet, as

540 the presence of moisture leads to higher heat losses from the skin (and thus colder sensations),

541 due to a higher thermal conductivity of a wet as opposed to a dry fabric (41). As for the same 
542 physical process (i.e. higher rate of heat losses), a cold-humid environment is perceived to be 543 colder than a cold-dry one (45).

544 Habituation factors could also explain the observed regional pattern in wetness perception.

545 As we are not provided with hygroreceptors (13), if we assume that, based on the concept of 546 perceptual learning (46), we learn to perceive cutaneous wetness, it would be reasonable to

547 hypothesize that the body regions more sensitive to skin wetness are the ones in which we are

548 more used to experience high levels of physical wetness, e.g. due to sweating. The outcomes

549 of this study could support this behavioral hypothesis. In the present study the back torso, and 550 particularly the lower back, a region which has been repeatedly shown to present some of the 551 highest levels of sweat production $(52,53)$, was indeed observed to be the most sensitive 552 region to wetness across torso.

554 Role of the thermal state of the body and the affective component of thermal stimulation

555 The cutaneous wetness perception was observed to be slightly higher under warm than under 556 thermo-neutral environmental conditions. As the thermosensitivity to cold was on the 557 contrary found to be slightly lower during the warm environmental condition, the increase in 558 overall wetness perception in the warm environment is more likely to be related to an 559 expectation effect (i.e. participants might have expected to sweat under the warm exposure) 560 than to a central sensory modulation of this perception. It could be argued that a higher level 561 of whole-body wetness, which might have influenced the way the cold-dry stimulus was 562 perceived locally on the skin (22), occurred during the warm exposure. However, as the 563 baseline wetness perceptions recorded pre-stimulation did not differ between the thermo564 neutral and the warm environmental exposures, and due to the resting condition of the 565 participants, it is unlikely that a higher level of whole-body wetness occurred or was 566 perceived by the participants. Nevertheless, the possibility to measure the skin's local 
567 hydration status should be considered in future studies, in order to investigate whether a

568 swelling state of the skin (due to sweat production) can affect the regional perception of skin 569 wetness (26).

570 With regards to the affective component of thermal stimulation, it deserves mention that the

571 local cold-dry stimulation of the torso was overall perceived as being unpleasant and that the

572 level of displeasure experienced varied significantly by location of stimulation. Interestingly,

573 the topographical distribution of the displeasure resulting from local thermal stimulation

574 corresponded to the regional distribution of cutaneous thermal and wetness perception

575 (compare fig. $2 D$ with $2 B$ and $2 C$ ). In this respect, it was observed that regions with a higher

576 thermosensitivity to cold and a higher frequency of wetness (e.g. the lower back, lateral mid-

577 back and medial upper back, as well as the lateral abdomen) were the ones in which the

578 application of the stimulus resulted as the most unpleasant (see fig. $3 B$ and $3 C$ ). These

579 outcomes confirm the physiological bases of pleasure $(9,10)$, particularly in the context of

580 thermal sensation and comfort (8).

581 It has been previously suggested that the hedonic attribute of a thermal stimulus is dependent

582 on the perception of the actual thermal state of the body: if the direction of the thermal

583 stimulus is oriented towards a shift in the thermal state of the body from its natural

584 homeostasis, then this will result in thermally unpleasant sensations; on the contrary, if the

585 direction of thermal stimulus is towards a re-establishment of the thermal state to its set point,

586 then this will result in thermally pleasant sensations (4). This concept, known as alliesthesia

587 (9), underpins the reason why a cold stimulus applied on normothermic individuals might be

588 perceived as more unpleasant than if the same was applied on hyperthermic individuals. As

589 during our experimental conditions participants were not expected to become hyperthermic

590 (due to resting conditions and short exposure duration), it is therefore clear why the

591 application of the cold stimulus was overall perceived as unpleasant. However, the novelty of 
592 this study is to provide a detailed topographical distribution of the regions of the torso in 593 which the exposure to cold stimuli might have a greater influence on the overall thermal 594 displeasure and discomfort. The fact that the back as well as the lateral abdomen presented a 595 higher sensitivity to thermal displeasure further our understanding of the role of the torso's 596 thermal comfort in the whole-body thermal comfort. Nakamura et al. $(2008,2013)(39,40)$ 597 have repeatedly shown that humans prefer a warm trunk and that abdominal cooling is often 598 perceived as more unpleasant than other regions' cooling. This is in line with the findings of 599 the present study, in which e.g. we observed the lateral abdomen to be amongst the regions in 600 which the application of the cold-dry stimulus was perceived as the most uncomfortable. As 601 local cooling of the abdomen has been shown to induce vasoconstriction of the corresponding 602 gastrointestinal tract, which in turn could affect the organ's function (33), it is therefore 603 reasonable to hypothesize that the higher sensitivity to thermal displeasure of this region 604 might represent a form of thermal protection aiming to maintain homeostasis (40).

605 It has to be acknowledged that, with regards to linking the changes in the internal state of the 606 body with the affective component of local thermal stimulation of the torso, the absence of a 607 direct measurement of core temperature represents a limitation of the current study. It could 608 be indeed speculated that, despite an increase in core temperature is unlikely to have occurred 609 within the experimental conditions of this study, a potential (although slight) fall in this value 610 could have occurred during the thermo-neutral exposure (due to the resting and semi-nude 611 conditions of the participants). Therefore, the contribution of even small changes in core 612 temperature to the overall hedonic component of thermal stimulation cannot be ruled out 613 conclusively. Nevertheless, the outcomes of this study further our understanding of the role 614 of cutaneous thermal afferents (as opposed to deep body) in influencing the hedonic attribute 615 of tactile stimulations. Recent evidence on the neurophysiology of affective touch have 616 indeed indicated that, apart from the role of core temperature, the presence of a particular 
617 class of cutaneous nerve fibers i.e. C-tactile afferents, which are specifically tuned to

618 affective as opposed to discriminative touch, could also play a significant role in influencing

619 the affective component of local thermal stimulation (1). In a recent study in which stroking-

620 like stimuli at 3 different temperatures [i.e. warm, neutral (same as skin temperature) and

621 cold)] were applied on participants' skin, Ackerley et al. (2014) (1) have shown that stimuli

622 with temperatures which deviated from neutrality (i.e. warm and cold) were perceived as less

623 pleasant than thermo-neutral stimuli. The authors concluded that the activity and role of C-

624 Tactile fibers in contributing to the hedonic component of tactile stimuli seems therefore to

625 be specifically tuned to the neutral temperature of a skin-stroking caress (1). These

626 observations seem supporting the results of the present study, in which we have demonstrated

627 that the further the stimuli deviated from thermo-neutrality (i.e. colder sensations), the greater

628 the displeasure experienced by the participants (see fig. $3 C$ ). Therefore, our findings indicate

629 that, despite the importance of monitoring core temperature, taking into account the potential

630 contributions of cutaneous C-Tactile afferents should also be considered in future

631 investigations as these could play a role in the hedonic component of local thermal

632 stimulation.

633 In conclusion, the present study found that cutaneous wetness perception varies significantly

634 across the human torso. We found that the existence of regional differences in cutaneous

635 thermosensitivity to cold translates into significant regional differences in cutaneous wetness

636 perception: regions with a high thermosensitivity to cold (e.g. the lower and lateral abdomen

637 and back) present skin wetness perceptions in greater magnitude and frequency. Also, it was

638 found that the regional distribution of cutaneous thermal and wetness perception was matched

639 by regional differences in the level of displeasure resulting from local thermal stimulation:

640 regions with a higher thermal and wetness perception (e.g. the lower and lateral abdomen and

641 back) present higher sensitivity to thermal displeasure. The outcomes of this study have a 
642 fundamental, clinical as well as an applied significance. From a fundamental point of view,

643 these indicate that cutaneous thermal, wetness and pleasantness sensations do not depend

644 solely on regional variations in $\mathrm{T}_{\text {sk }}$ but also on an uneven weighting and integration of

645 peripheral thermoafferent information which could be influenced by behavioral and

646 habituation factors. From a clinical point of view, due to a recent interest in mapping bodily

647 sensations such as pain (35), the body maps of torso thermal, wetness and pleasantness

648 sensation developed in this study could be used as a frame of reference for normal and altered

649 somatosensory function in the context of multiple sclerosis or polyneuropathies, diseases

650 which are usually accompanied by alteration of normal somatosensory function $(30,42,47$,

651 56). Finally, from an applied point of view, these body maps could be useful in improving the

652 design of protective clothing in order to optimize thermal protection and maximize thermal

653 comfort under extreme environmental conditions (e.g. cold air/water exposures).

654

655 Role of the funding source

656 The present research was done in the context of an industry co-funded Ph.D. Loughborough

657 University and Oxylane Research provided financial support.

658 References

659 1. Ackerley R, Backlund Wasling H, Liljencrantz J, Olausson H, Johnson RD, 660 Wessberg, J (2014). Human C-Tactile afferents are tuned to the temperature of a skin661

662

663

664

665

666

667

668

669

670

671 stroking caress. J Neurosci 34: 2879-2883.

2. Ackerley R, Olausson H, Wessberg J, McGlone F. Wetness perception across body sites. Neurosci Lett 522: 73-77, 2012.

3. Acock A. A gentle introduction to stata. Texas: Stata Press, 2010.

4. Attia M, Engel P. Thermal pleasantness sensation: an indicator of thermal stress. Eur $J$ Appl Physiol 50: 55-70, 1982.

672

5. Auliciems A. Thermal sensation and cell adaptability. Int J Biometeorol 58:325-35, 2014. 
6. Bergmann Tiest WM, Kosters ND, Kappers AML, Daanen HAM. Haptic perception of wetness. Acta Psychol (Amst) 141: 159-163, 2012.

675

676

677

678

679

680

681

682

683

684

685

686

687

688

689

690

691

692

693

694

695

696

697

698

699

700

701

702

703

704

705

706

707

708

709

710

711

712

713

714

715

716

7. Burke W, Mekjavic I. Estimation of regional cutaneous cold sensitivity by analysis of the gasping response. J Appl Physiol (1985) 71:1933-1940, 1991.

8. Cabanac M, Massonnet B, Belaiche R. Preferred skin temperature as a function of internal and mean skin temperature. J Appl Physiol 33: 699-703, 1972.

9. Cabanac M. Physiological role of pleasure. Science 173: 1103-1107, 1971.

10. Cabanac M. Pleasure: the common currency. J Theor Biol 155: 173-200, 1992.

11. Campero M, Serra J, Bostock H, Ochoa JL. Slowly conducting afferents activated by innocuous low temperature in human skin. J Physiol 535: 855-865, 2001.

12. Candas V, Libert J, Vogt J. Human skin wettedness and evaporative efficiency of sweating. J Appl Physiol Respir Environ Exerc Physiol 46: 522-528, 1979.

13. Clark R, Edholm O. Man and his thermal environment. London : E. Arnold, 1985.

14. Claus D, Hilz M. Methods of measurement of thermal thresholds. Acta Neurol Scand 76: 288-96, 1987.

15. Craig A, Chen K, Bandy D, Reiman E. Thermosensory activation of insular cortex. Nat Neurosci 3: 184-190, 2000.

16. Daanen HAM. Method and system for alerting the occurrence of wetness. EP Patent $2,110,1082009$.

17. Driver J, Spence C. Multisensory perception: Beyond modularity and convergence. Curr Biol 10: R731-R735, 2000.

18. Filingeri D, Fournet D, Hodder S, Havenith G. Why wet feels wet? A neurophysiological model of human cutaneous wetness sensitivity. J Neurophysiol (June 18, 2014). doi: 10.1152/jn.00120.2014.

19. Filingeri D, Redortier B, Hodder S, Havenith G. The role of decreasing contact temperatures and skin cooling in the perception of skin wetness. Neurosci Lett 551: 65-69, 2013.

20. Filingeri D, Redortier B, Hodder S, Havenith G. Thermal and tactile interactions in the perception of local skin wetness at rest and during exercise in thermo-neutral and warm environments. Neuroscience 258: 121-130, 2014. 
21. Filingeri D, Redortier B, Hodder S, Havenith G. Warm temperature stimulus suppresses the perception of skin wetness during initial contact with a wet surface. Skin Res Technol (February 14, 2014). doi: 10.1111/srt.12148, 2014.

22. Fukazawa T, Havenith G. Differences in comfort perception in relation to local and whole body skin wettedness. Eur J Appl Physiol 106: 15-24, 2009.

23. Gallio M, Ofstad TA, Macpherson LJ, Wang JW, Zuker CS. The coding of temperature in the Drosophila brain. Cell 144: 614-24, 2011.

24. Gan Y, Liu Y, Ding X, Pan N. Thermal sensation at index finger while applying external pressure at upper arm. J Therm Biol 37: 502-509, 2012.

25. Garn SM. Types and distribution of the hair in man. Ann N Y Acad Sci 53: 498-507, 1951.

26. Gerrett N, Redortier B, Voelcker T, Havenith G. A comparison of galvanic skin conductance and skin wettedness as indicators of thermal discomfort during moderate and high metabolic rates. $J$ Therm Biol 38: 530-538, 2013.

27. Gordon NF, Pescatello LS. ACSM's guidelines for exercise testing and prescription. Philadelphia: Lippincott Williams \& Wilkins, 2010.

28. Haggard P, Iannetti GD, Longo MR. Spatial sensory organization and body representation in pain perception. Curr Biol 23: R164-R176, 2013.

29. Houdas Y, Ring E. Human body temperature-Its measurement and regulation. New York: Plenum Press, 1982.

30. Hulse R, Wynick D, Donaldson LF. Intact cutaneous C fibre afferent properties in mechanical and cold neuropathic allodynia. Eur J Pain 14: 565.e1-565.e10, 2010.

31. Keatinge W, Nadel J. Immediate respiratory response to sudden cooling of the skin. $J$ Appl Physiol 20: 65-69, 1965.

32. Kondo N, Nakadome M, Zhang K, Shiojiri T, Shibasaki M, Hirata K, Iwata A. The effect of change in skin temperature due to evaporative cooling on sweating response during exercise. Int J Biometeorol 40: 99-102, 1997.

33. Kuntz A, Haselwood LA. Circulatory reactions in the gastrointestinal tract elicited by localized cutaneous stimulation. Am Heart J 20: 743-749, 1940.

759 
774

775

776

777

778

779

780

781

782

783

784

785

786

787

788

789

790

791

792

793

794

795

796

797

798

799

800

801

802

803

34. Lee J, Nakao K, Tochihara Y. Validity of perceived skin wettedness mapping to evaluate heat strain. Eur J Appl Physiol 111: 2581-2591, 2011.

35. Mancini F, Bauleo A, Cole J, Lui F, Porro CA, Haggard P, Iannetti GD. Whole-body mapping of spatial acuity for pain and touch. Ann Neurol doi: 10.1002/ana.24179, 2014.

36. McGlone F, Reilly D. The cutaneous sensory system. Neurosci Biobehav Rev 34: 148$159,2010$.

37. Montell C. TRP Channels: It's Not the Heat, It's the Humidity. Curr Biol 18: R123-R126, 2008.

38. Nadel ER, Stolwijk JA. Effect of skin wettedness on sweat gland response. $J$ Appl Physiol 35: 689-694, 1973.

39. Nakamura M, Yoda T, Crawshaw L, Yasuhara S, Saito Y, Kasuga M, Nagashima K, Kanosue K. Regional differences in temperature sensation and thermal comfort in humans. J Appl Physiol (1985) 105: 1897-1906, 2008.

40. Nakamura M, Yoda T, Crawshaw LI, Kasuga M, Uchida Y, Tokizawa K, Nagashima K, Kanosue K. Relative importance of different surface regions for thermal comfort in humans. Eur J Appl Physiol 113: 63-76, 2013.

41. Niedermann R, Rossi R. Objective and subjective evaluation of the human thermal sensation of wet fabrics. Text Res J 82: 374-384, 2012.

42. Nolano M, Provitera V, Estraneo A, Selim MM, Caporaso G, Stancanelli A, Saltalamacchia AM, Lanzillo B, Santoro L. Sensory deficit in Parkinson's disease: evidence of a cutaneous denervation. Brain 131: 1903-1911, 2008.

43. Olesen B, Brager G. A better way to predict comfort: The new ASHRAE standard 552004. ASHRAE J 8: 20-26, 2004.

44. Ouzzahra Y, Havenith G, Redortier B. Regional distribution of thermal sensitivity to cold at rest and during mild exercise in males. J Therm Biol 37: 517-523, 2012.

45. Plante a. M, Holcombe BV, Stephens LG. Fiber hygroscopicity and perceptions of dampness: Part I: subjective trials. Text Res J 65: 293-298, 1995.

46. Pleger B, Villringer A. The human somatosensory system: from perception to decision making. Prog Neurobiol 103: 76-97, 2013.

47. Rae-Grant AD, Eckert NJ, Bartz S, Reed JF. Sensory symptoms of multiple sclerosis: a hidden reservoir of morbidity. Mult Scler 5: 179-183, 1999. 
48. Schepers R, Ringkamp M. Thermoreceptors and thermosensitive afferents. Neurosci Biobehav Rev 34: 177-184, 2010.

807

808

809

810

811

812

813

814

815

816

817

818

819

820

821

822

823

824

825

826

827

828

829

830

831

832

833

834

835

836

837

838

839

840

841

842

843

844

845

846
49. Schlader Z, Stannard S, Mündel T. Human thermoregulatory behavior during rest and exercise - a prospective review. Physiol Behav 99: 269-275, 2010.

50. Schlader ZJ, Perry BG, Jusoh MRC, Hodges LD, Stannard SR, Mündel T. Human temperature regulation when given the opportunity to behave. Eur J Appl Physiol 113: 1291-1301, 2013.

51. Sendowski I, Savourey G, Launay J, Besnard Y, Cottet-Emard J, Pequignot J, Bittel J. Sympathetic stimulation induced by hand cooling alters cold-induced vasodilatation in humans. Eur J Appl Physiol 81: 303-309, 2000.

52. Smith CJ, Havenith G. Body mapping of sweating patterns in male athletes in mild exercise-induced hyperthermia. Eur J Appl Physiol 111: 1391-1404, 2011.

53. Smith CJ, Havenith G. Body Mapping of Sweating Patterns in Athletes: A Sex Comparison. Med Sci Sports Exerc 44: 2350-2361, 2012.

54. Spray DC. Cutaneous temperature receptors. Annu revi physiol 48: 625-638, 1986.

55. Strusberg I, Mendelberg RC, Serra HA, Strusberg AM. Influence of weather conditions on rheumatic pain. J Rheumatol 29: 335-338, 2002.

56. Susser E, Sprecher E, Yarnitsky D. Paradoxical heat sensation in healthy subjects: peripherally conducted by A delta or C fibres? Brain 122: 239-246, 1999.

57. Tichy H, Kallina W. Insect hygroreceptor responses to continuous changes in humidity and air pressure. J Neurophysiol 103: 3274-3286, 2010.

58. Yildiz BO, Bolour S, Woods K, Moore A, Azziz R. Visually scoring hirsutism. Hum Reprod Update 16: 51-64, 2010. 
847 Figure 1: Name and exact anatomical locations of the 12 skin sites targeted for stimulation.

848

849 Figure 2: Body maps showing the regional distribution of (A) skin cooling $\left({ }^{\circ} \mathrm{C}\right),(\mathrm{B})$ absolute 850 mean votes for thermal sensation, (C) frequency of wetness perception and (D) absolute mean 851 votes for pleasantness sensation, as a result of the $10 \mathrm{~s}$ application of the relative cold-dry 852 stimulus $\left(15^{\circ} \mathrm{C}\right.$ lower than local $\left.\mathrm{T}_{\text {sk }}\right)$ to each skin site, collapsed over all conditions. Data 853 were collected on the left side of the body and the body maps presented were developed 854 assuming left-right symmetry (see Ouzzahra et al., 2012) (44). Regions showing greater skin 855 cooling, colder sensations, more frequent wetness perceptions and more unpleasant 856 sensations are represented in darker colors. The rating scales used by the participants to score 857 their absolute thermal and pleasantness sensations are reported next to the respective body 858 maps. Two main tendencies are shown. First, the regional differences in thermal, wetness and 859 pleasantness sensation present a similar pattern across the torso (e.g. as opposed to the chest, 860 the lateral and lower back appears more sensitive to cold, wetness and thermal displeasure). 861 Second, these sensory patterns seem independent from the regional variations in skin cooling 862 (i.e. regions which show greater skin cooling, such as the lateral chest, are not necessarily the 863 ones in which the stimulus was perceived as colder, more often wet or more unpleasant).

864 Figure 3: Relationship between: (A) thermal (cold) sensation and the frequency of perceived 865 wetness; (B) pleasantness sensation and the frequency of perceived wetness; (C) thermal 866 (cold) sensation and pleasantness sensation. Data are reported as mean for each skin site, 867 collapsed over all conditions, and standard deviation (horizontal and vertical lines). There is a 868 highly significant correlation between the level of coldness experienced and the frequency of 869 perceived wetness (i.e. increasing coldness and increasing wetness), the level of pleasure 870 experienced and the frequency of perceived wetness (i.e. decreasing pleasantness and 
871 increasing wetness), and the level of coldness and pleasure experienced (i.e. increasing 872 coldness and decreasing pleasantness).

873 Table 1: Significance levels of the multiple comparisons for the 12 skin sites are reported for 874 skin cooling $\left(\Delta \mathrm{T}_{\mathrm{sk}}\right)$, thermal (TS), wetness (WP) and pleasantness (PS) sensation.

875 Table 1 footnote: ${ }^{*} p<0.05 ; \uparrow p<0.01 ; \uparrow p<0.001$. 
1. Medial chest

( $270 \mathrm{~mm}$ above the umbilicus)

\section{Lateral chest}

(70 $\mathrm{mm}$ above the nipple)

3. Medial upper abdomen (130 $\mathrm{mm}$ above the umbilicus)

\section{Lateral upper abdomen}

(30 $\mathrm{mm}$ below the nipple)

5. Medial abdomen

(30 $\mathrm{mm}$ above the umbilicus)

6. Lateral lower abdomen (45 $\mathrm{mm}$ above the anterior superior iliac crest)

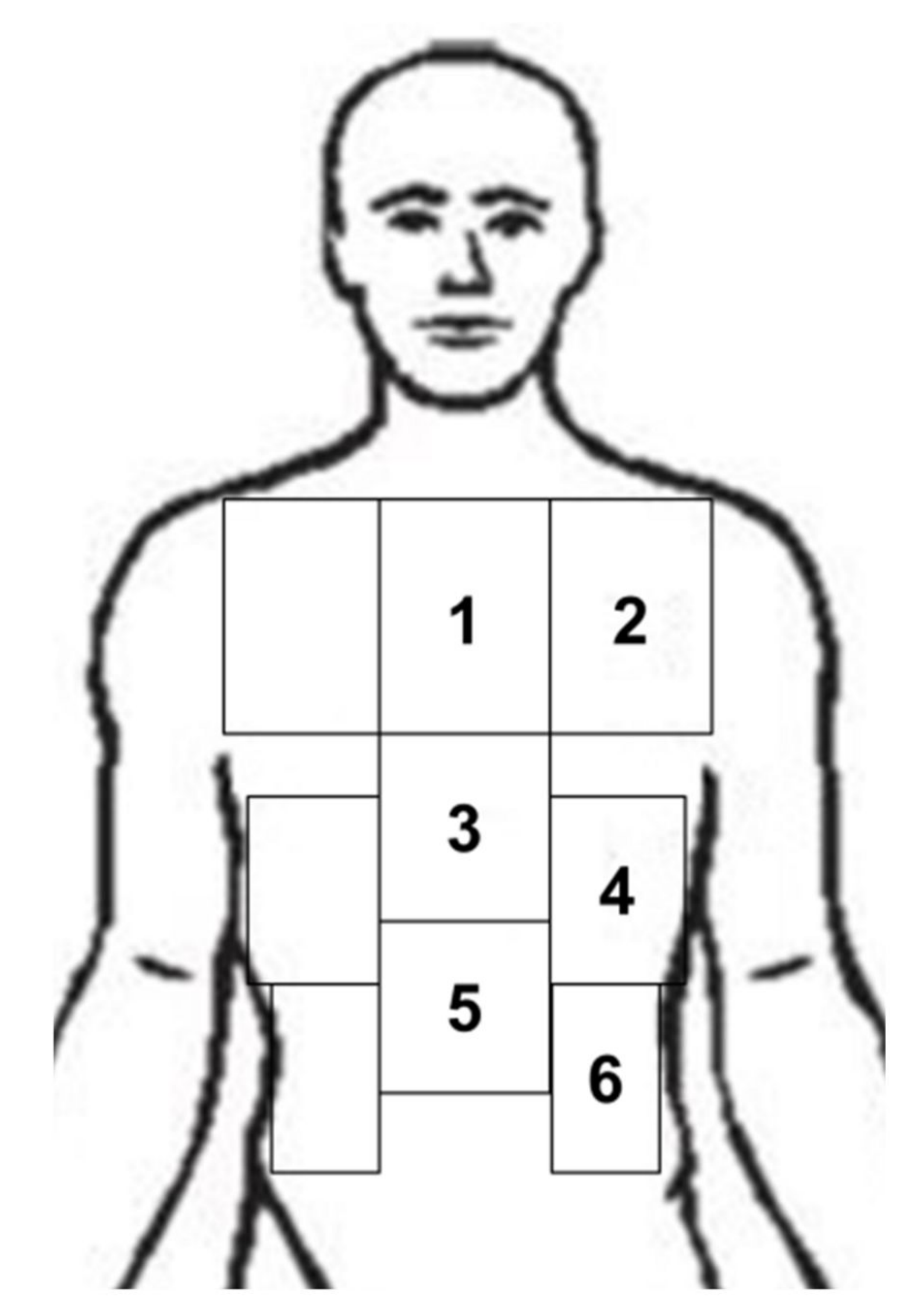

7. Lateral upper back (50 $\mathrm{mm}$ above the inferior angle of the scapula)

\section{Medial upper back}

(30 mm medial to 7)

\section{Lateral mid-back}

(10 $\mathrm{mm}$ below the inferior angle of the scapula)

\section{Medial mid-back}

(30 mm medial to 9)

11. Lateral lower back

(165 mm below the inferior angle of the scapula)

12. Medial lower back

(30 mm medial to 11) 

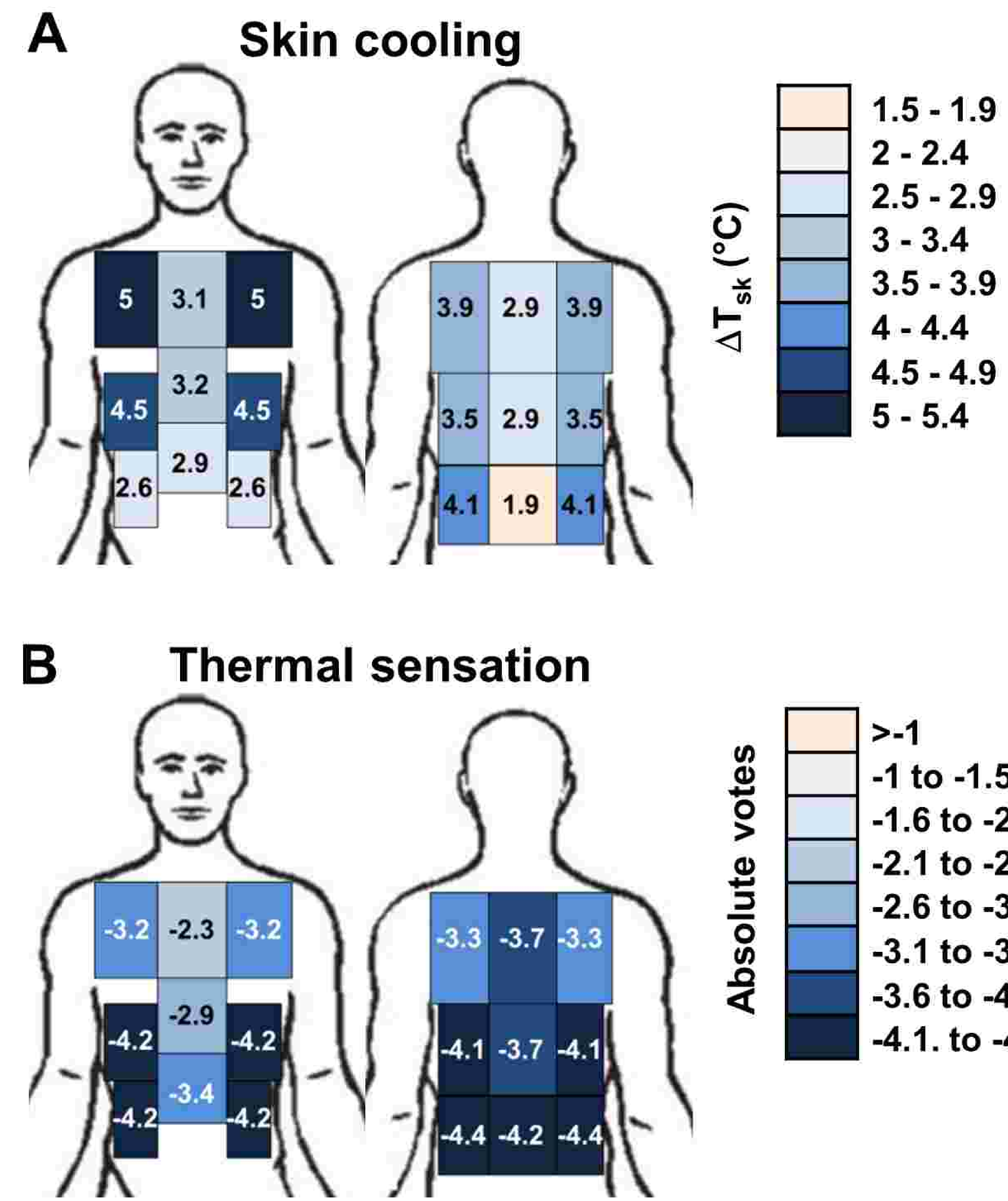

\section{Thermal scale \\ 0 Neutral \\ -2 Slightly cool \\ -6 Very cold}

$>-1+4$ Warm -1 to $-1.5+3$

-1.6 to -2

$+2$

-2.1 to -2.5

-2.6 to -3

-3.1 to -3.5

-3.6 to -4

-4.1 . to -4.5

$+1$

$-1$

$-3$

-4 Cold

$-5$

C Frequency of wetness
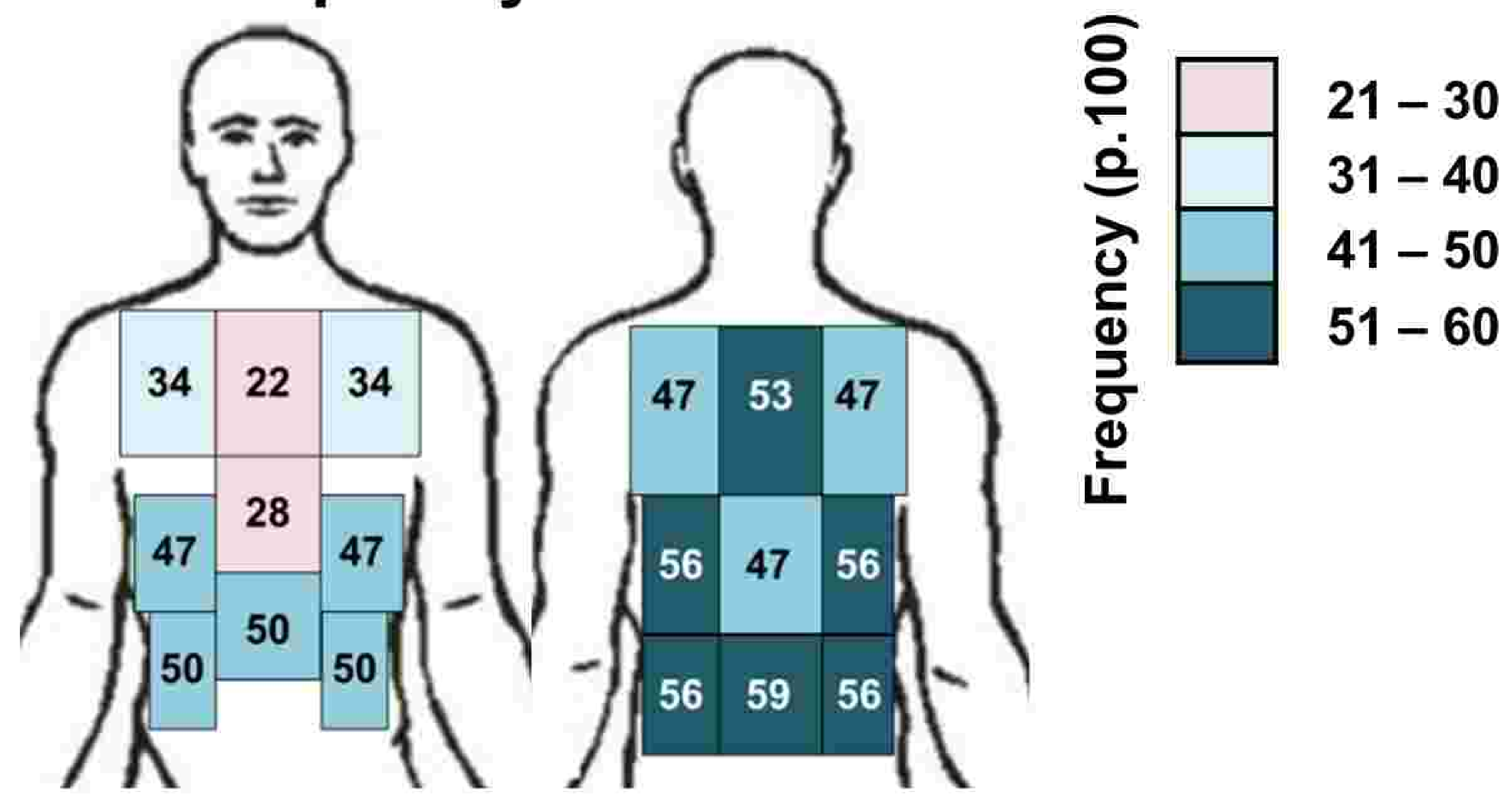

D Pleasantness sensation


Pleasantness scale

+4 Pleasant

$+3$

2 Slightly pleasant

$+1$

0 Neutral

-2 Slightly unpleasant

$-3$

-6 Very unpleasant 


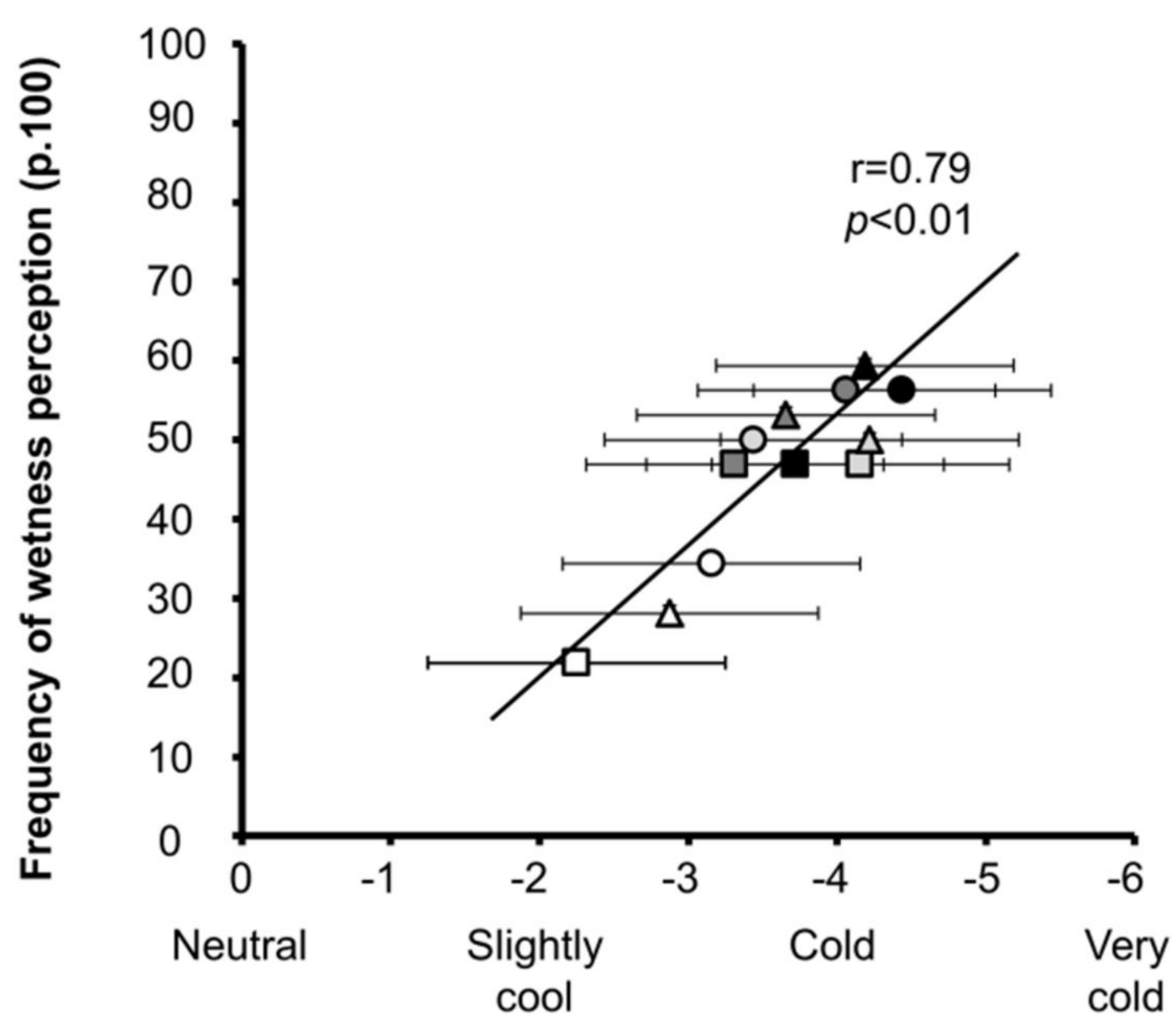

口Medial chest

OLateral chest

$\Delta$ Medial upper abdomen 口Lateral upper abdomen OMedial abdomen

$\Delta$ Lateral lower abdomen

口Lateral upper back

$\Delta$ Medial upper back

OLateral mid-back

-Medial mid-back

- Lateral lower back

$\Delta$ Medial lower back

\section{Thermal sensation scale}

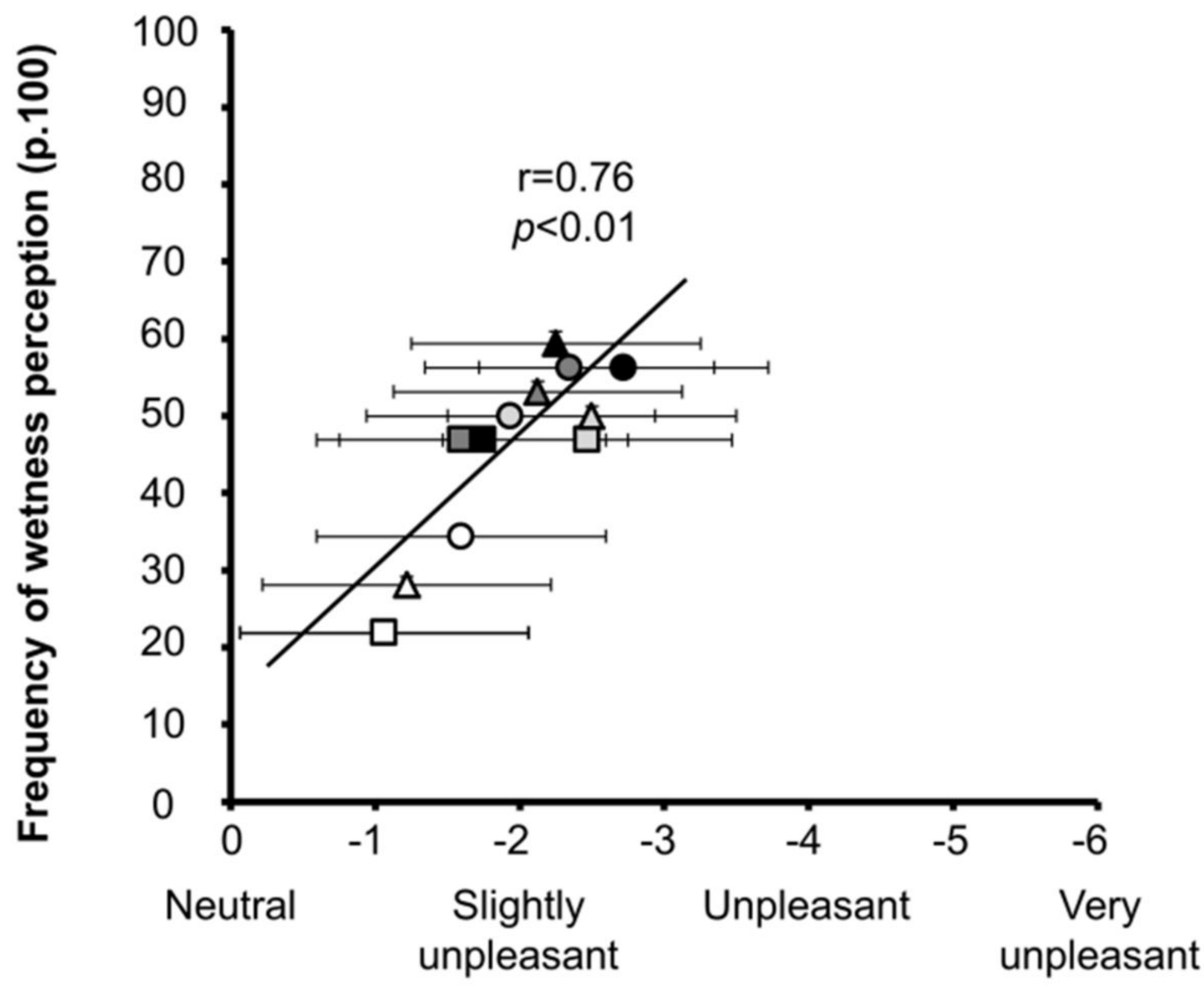

Pleasantness sensation scale

C

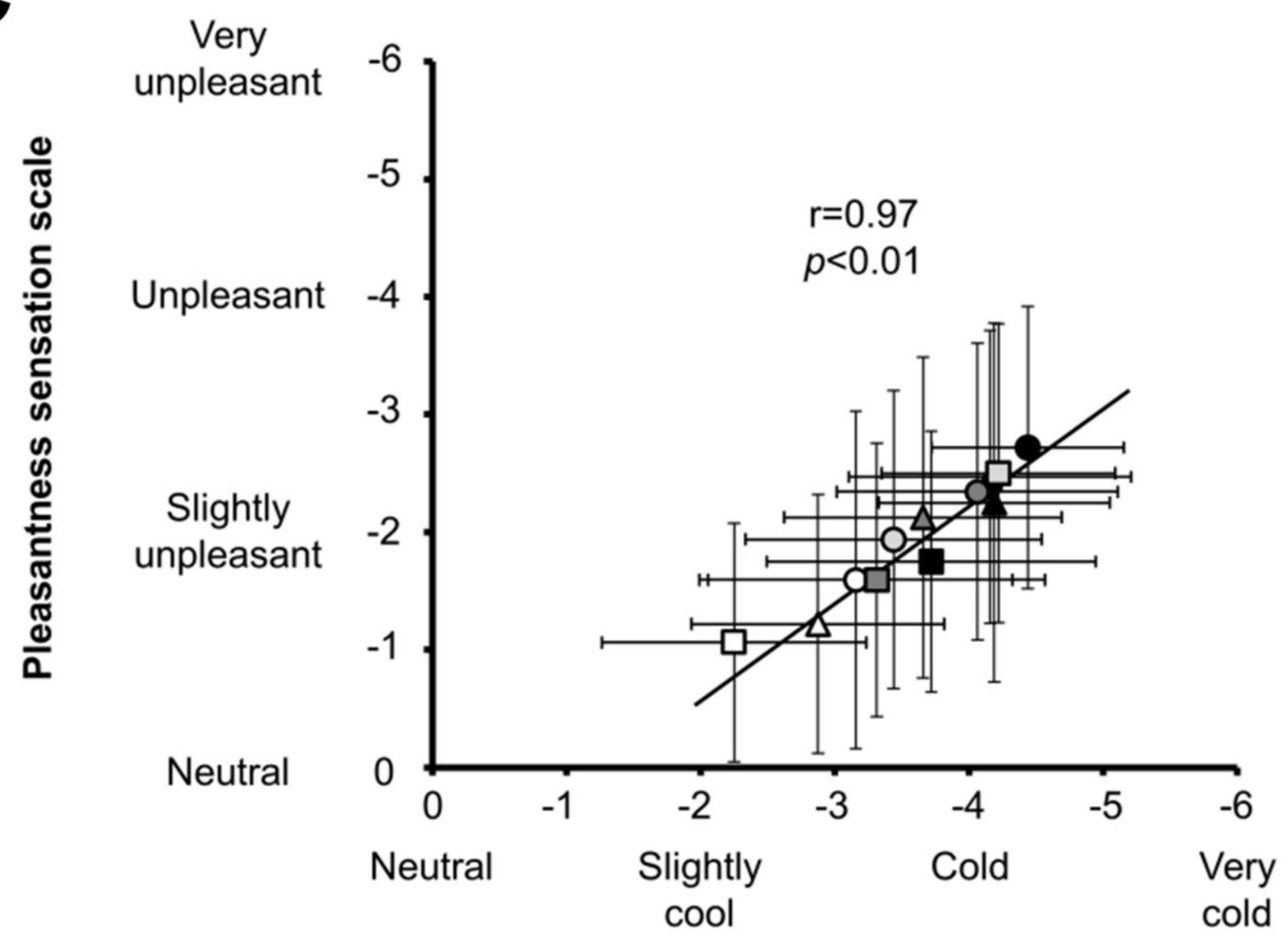

Thermal sensation scale 


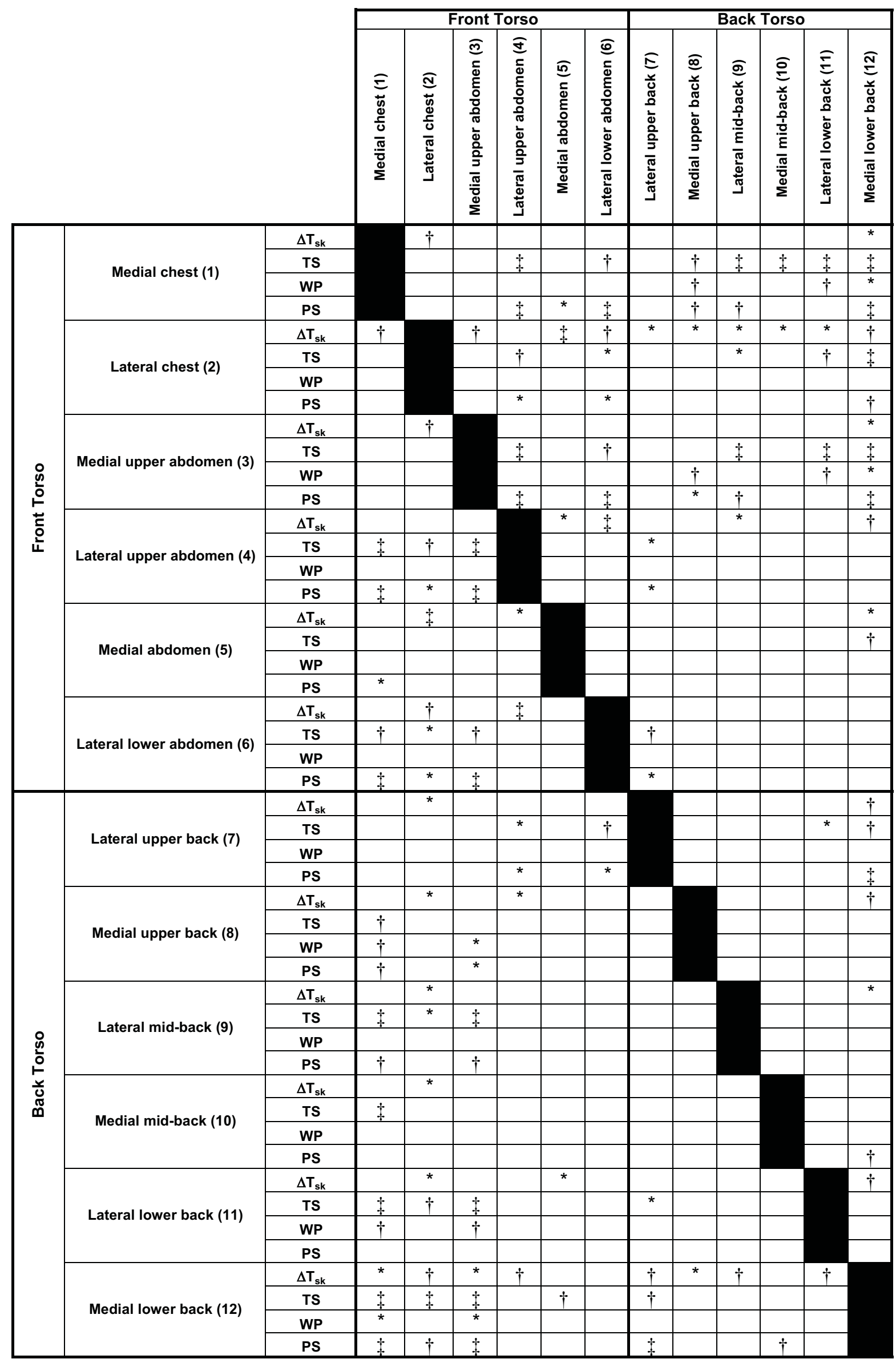

